\title{
A Switching-Based Interference Control for Booster Separation of Hypersonic Vehicle
}

\author{
Qin Zhong $\mathbb{D}^{1}$ and Wenbin $\mathrm{Wu} \mathbb{D}^{2}$ \\ ${ }^{1}$ School of Astronautics, Northwestern Polytechnical University, Xi'an, China \\ ${ }^{2}$ Institute of Modern Control Technology, Xi'an, China \\ Correspondence should be addressed to Qin Zhong; zhongqin_nwpu@126.com
}

Received 25 October 2021; Revised 17 November 2021; Accepted 19 November 2021; Published 15 December 2021

Academic Editor: Narasimhan Venkateswaran

Copyright (C) 2021 Qin Zhong and Wenbin Wu. This is an open access article distributed under the Creative Commons Attribution License, which permits unrestricted use, distribution, and reproduction in any medium, provided the original work is properly cited.

\begin{abstract}
Whether launching from the ground or in the air, hypersonic vehicles need the booster to accelerate to a predetermined window, so as to meet the requirements of scramjet engine ignition. Therefore, there is interference suppression between boosters and hypersonic vehicles under the high dynamic pressure, which has become a key technical problem that affects the success of flight tests, especially when the aircraft is statically unstable. A method of variable structure switching-based control is proposed in this paper for rapid suppression on hypersonic vehicle booster separation interference. Switching control systems in real time according to state changes caused by flow field interference, the method can keep the attitude stability of hypersonic vehicle booster separation under the high dynamic pressure of static instability. The aerodynamic calculation model of the hypersonic vehicle booster separation process is established first, which adopts an unsteady solution and clarifies the aerodynamic interference characteristics of the afterbody on the vehicle in booster separation. Then, according to the characteristics of the flow field, the dynamics of the vehicle in and out of the interference area are converted into subsystems with switching characteristics. Using the dimension reduction and variable structure method, the switching control surface of the control system is established. On the basis of the vehicle state changes caused by flow field, the control system on the orbital change surface can be switched in real time to achieve stable attitude in the process of separation interference. Meanwhile, considering the additional interference torque generated by the afterbody to the vehicle in the separation process, a control system for interference suppression of the booster separation is designed. Simulation results verify that the designed control system can rapidly suppress the booster separation interference when the dynamic pressure is about $150 \mathrm{kPa}$ and the vehicle has the static instability of $5 \%$, thereby realizing the stable attitude of the vehicle.
\end{abstract}

\section{Introduction}

The near space air-breathing hypersonic vehicle must have certain height, speed, and dynamic pressure, only in this way can the scramjet engine be reliably ignited. Whether launching in the air or from the ground, there is a process of accelerating to scramjet ignition window at low speed. Then, the booster is separated from the vehicle, the scramjet is ignited, and the hypersonic vehicle flies autonomously. The scramjet can be ignited at a relatively high speed and under high dynamic pressure; therefore, the external flow field will generate strong interference to the hypersonic air- craft in its stage separation process. When the vehicle is statically stable, the control system can control it again after the vehicle flies out of the interference flow field. In this process, the separation interference only affects the vehicle attitude and has no mutual coupling effect with the control system. However, with the development of the weaponization of vehicles, hypersonic vehicles increasingly present larger characteristics of static instability. After stage separation, the vehicle control system must be activated to control the statically unstable projectile body in the interference flow field area. Therefore, the stage separation interference flow field will not only affect the attitude of the projectile body 
but also interact with the control system and even show the steerage reversal, which causes the control divergence and the instability of vehicle attitude. The stage separation interference under the complex flow field with large dynamic pressure poses a great challenge to the design of the statically unstable hypersonic vehicle control system.

The stage separation of hypersonic vehicles is regarded by NASA as one of the two key problems in the X-43A hypersonic flight test, attracting extensive attention of scholars. The literature [1-5] studied the feasibility of X43A stage separation and the aerodynamic characteristics of separation. Shengjiang and Chaoyi [6] elaborated on the stage separation strategy and flight test verification of $\mathrm{X}$ 43A hypersonic vehicle. Using the linear interpolation method, Li Yongtao and Xiangju [7] studied the aerodynamic characteristics of the stage separation and the stage separation problem of the X-43A hypersonic vehicle.

Aiming at the stage separation of hypersonic vehicles, six degrees of freedom solver and local mesh reconstruction based on a nonstructured mesh method were proposed to stimulate the separation process. Based on this method, Xun et al. [8] studied the complex flow field interference between the precursor discharge and the afterbody resistance plate. Results included the development rule of the motion parameters and aerodynamic parameters of the vehicle and the booster in the separation process under the cold separation state of weak perturbance. Li et al. [9] studied the rocket stage transition process by changing the separation conditions and obtained the difference of flow field distribution between stages at different separation distances. Binbin et al. [10] proposed a rudder deflection presetting design method based on the CMAC neural network for aerodynamic interference caused by hypersonic vehicle stage separation. However, this method needs to rely on the prior information of separation interference.

The above-mentioned literatures studied the aerodynamic characteristics of the stage separation of the hypersonic vehicles and the safety of the separation. However, they considered neither the attitude changes between the frontbody and afterbody at the beginning of the separation nor the impact of the antishock wave generated by the afterbody on the steerage. The antishock wave generated by the booster afterbody will not only produce a local high pressure area at the tail of the aircraft, which interferes with the attitude of the vehicle, but also cause an antimanipulation effect on the steerage. If the control system suppresses the interference of the projectile in accordance with the normal operating efficiency at this time, the control system will make a positive feedback due to the counter-control of the control surface, thereby leading to its divergence.

The process of stage separation can be regarded as the anticontrol phase of the control surface under the antishock wave action and the normal control phase out of the interference zone. The traditional control system relying on the robustness of the control system only adopts a single control mode, which makes it difficult to realize reliable control of the above two phases.

Recently, sliding mode control methods have been used to study switching systems and many important conclusions have been achieved [11-13]. Among them, Wu and Lam [11] studied sliding mode control for a class of switched systems with time-delay. By performing a standard transformation on the system, a linear sliding mode surface was designed. The exponential stability of the sliding mode was analyzed by using the average dwell time method, and a sliding mode control law was designed to ensure that the state trajectory of the system drives to the sliding mode surface within a limited time. Then, the above results were further extended to random switching systems [12] and discretetime switching systems [13]. Aiming at a class of uncertain systems and considering the difference of input matrices, Liu Yonghui proposed an input matrix weighting method to construct a common sliding mode surface, designed state, and time-dependent switching signals, and proved its stability.

There is strong aerodynamic interference of the afterbody to the front body during the stage separation of the hypersonic vehicle booster, and the control system has a counter-effect on the control surface. To solve the above problems, nonquantitative local mesh reconstruction technology is adopted in this paper to calculate the flow field interference of the front body caused by the antishock wave generated by the afterbody in the stage separation process. A model of aerodynamic interference for hypersonic vehicle stage separation is established, and a method of hypersonic separation control based on sliding mode switching control is proposed.

According to the aerodynamic characteristics of hypersonic vehicle stage separation, this method regards the states of the aircrafts in and out of the interference area as two subsystems of the switching system. The sliding mode switching equation is constructed by using the state and time, and the sliding mode controller is designed to effectively ensure the safety of stage separation when there is complex aerodynamic interference during the stage separation of hypersonic vehicles. The simulation results verify that the designed control system can realize the reliable booster separation under the high dynamic pressure and make a good inhibiting effect on the interference in the separation process.

\section{Methodology}

2.1. Description of the Hypersonic Vehicle Stage Separation Process. The stage separation process between the tandem booster and the hypersonic vehicle is shown in Figure 1. When the hypersonic vehicle reaches the separation window, the attitudes of its front body and afterbody remain the same, and there is no mutual flow field interference. After the vehicle receives the stage separation instruction, the explosive bolt will be detonated, and the two bodies will be separated. Due to the impulse interference of the explosion bolt and the relative movement of the attitude between the precursors after separation, the attitude changes of the two bodies cause complex flow field change; especially, the rear body will generate antishock wave, which produces a torque to the front body. At the same time, the rudder surface control efficiency of the front body will be affected, resulting in antimanipulation. 


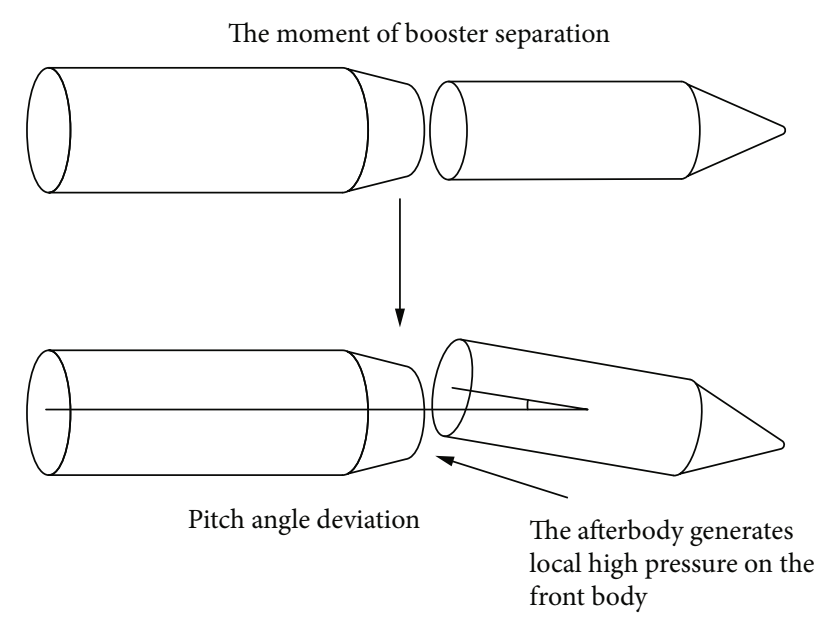

Figure 1: Schematic diagram of the hypersonic vehicle stage separation.

For stage separation, this paper adopts the local mesh reconstruction technology under nonstructured mesh, $\mathrm{CFD} / 6 \mathrm{DoF}$ coupling calculation, the six-degree-of-freedom unsteady method suitable for solving multibody separation problems with complex configurations, and the overlapping mesh technology to accelerate convergence.

The following are the main states of stage separation:

(a) Mach number: 5.6, altitude: $19 \mathrm{~km}$, angle of attack: $2.0^{\circ}$, sideslip angle: $0.0^{\circ}$, and separation: $200 \mathrm{~mm}$

(b) Mach number: 5.6, altitude: $19 \mathrm{~km}$, angle of attack: $0.0^{\circ}$, sideslip angle: $0.0^{\circ}$, and separation: $1900 \mathrm{~mm}$

Figures $2-5$ show the pressure nephogram of the fuselage during the booster separation process.

It can be seen from the figure that in the separation process, when the booster stage reverses injection engine works and the distance between stages is small, there will be a certain afterbody effect. The forward jet will produce a certain aerodynamic interference to the precursor aircraft and interact with the aircraft tail, thus affecting the stability of the precursor aircraft. In the process of interstage separation, the middle section is a high-pressure area, which makes the resistance of the aircraft decrease and the resistance of the booster increase. The aerodynamic effect will accelerate the process of interstage separation. Because the interstage separation studied in this paper is controlled by the reverse injection engine, the working of the reverse injection engine will have a certain aerodynamic interference impact on the attitude of the booster stage, forming a blocking effect on the incoming flow in front of the nozzle, resulting in a large range of high-pressure area, and a low-pressure area after the nozzle due to the injection of high-pressure air flow. Under the joint action of the two, the force and attitude of the first-class aircraft are affected.

Figure 6 shows the variation curve of the pitching moment coefficient of the hypersonic vehicle with the angle of attack and deflection angle of control surface during the separation process.

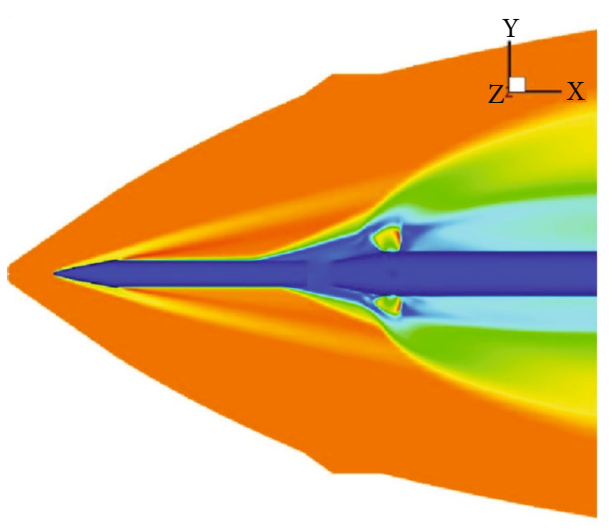

FIGURE 2: Mach nephogram in state a.

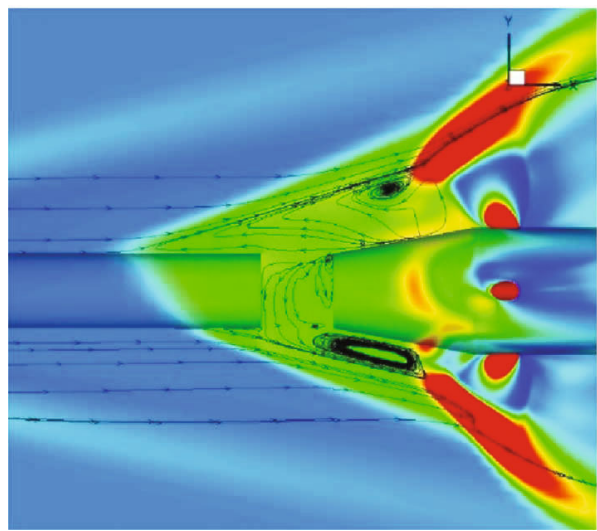

Figure 3: Fuselage pressure nephogram in state a.

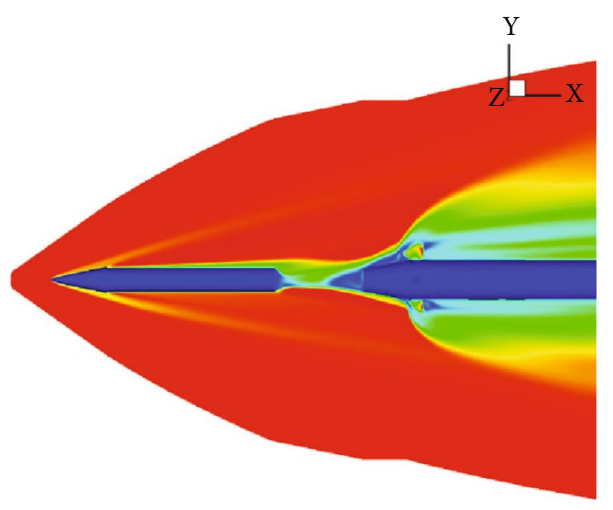

Figure 4: Mach nephogram in state b.

It can be clearly seen from Figures 6 and 7 that due to the influence of the interference flow field, the pitching moment coefficient of the vehicle decreases, and the steerage shows aileron reversal in the process of stage separation. During the boost separation of hypersonic vehicle, the tail wing of the vehicle is affected by the interference flow field, and the control effect of the control surface will be weakened. If the controller is designed based on the aerodynamic data as shown in Figure 6, the control system will diverge when the aerodynamic characteristics of the vehicle appear 


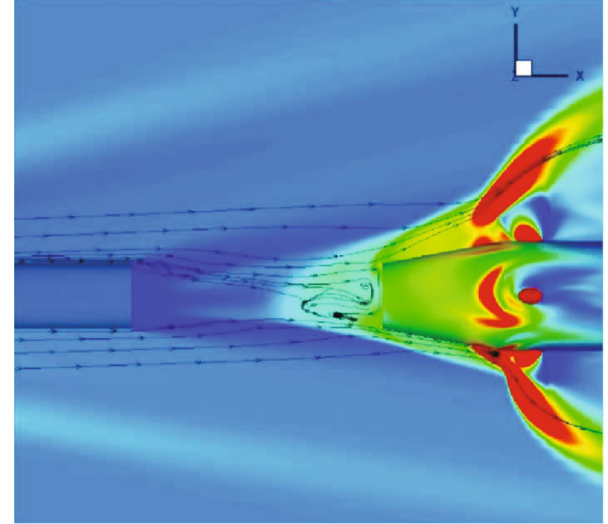

Figure 5: Fuselage pressure nephogram in state b.

anticontrol phenomenon under the influence of the interference flow field (as shown in Figure 7).

2.2. Dynamic Modeling of the Hypersonic Vehicle. Mainly considering the longitudinal motion of hypersonic aircraft, the dynamic model of the hypersonic vehicle in the process of stage separation is given below:

$$
\left\{\begin{array}{l}
m \frac{d V}{d t}=-X-m g \sin \theta, \\
J_{z} \frac{d \omega_{z}}{d t}=M_{z}, \\
\frac{d \vartheta}{d t}=\omega_{z}, \\
\vartheta=\theta+\alpha \\
X=\frac{1}{2} \rho V^{2} S C_{a}, \\
Y=\frac{1}{2} \rho V^{2} S C_{n}, \\
M_{z}=\frac{1}{2} \rho V^{2} S L C_{m_{z}},
\end{array}\right.
$$

where $\rho$ is the atmospheric density, $\bar{S}$ is the reference area, and $L$ is the reference length. The aerodynamic model of the longitudinal channel drag coefficient $C_{D}$, lift coefficient $C_{L}$, and pitching moment coefficient $C_{M_{y y}}$ on angle of attack, angle of pitch rudder deflection, and Mach number is

$$
\left\{\begin{array}{l}
\rho=\rho_{0} \exp \left(\frac{-\left(H-H_{0}\right)}{H_{s}}\right) \\
C_{D}=C_{D}^{0}+C_{D}^{\alpha} \alpha+C_{D}^{\delta_{e}} \delta_{e}+C_{D}^{M a} M a+C_{D}^{M a \cdot \alpha} M a \cdot \alpha+C_{D}^{M a \cdot \delta_{e}} M a \cdot \delta_{e}+C_{D}^{M a^{2}} M a^{2} \\
C_{L}=C_{L}^{0}+C_{L}^{\alpha} \alpha+C_{L}^{\delta_{e}} \delta_{e}+C_{L}^{M a} M a+C_{L}^{\alpha^{2}} \alpha^{2}+C_{L}^{\alpha \cdot \delta_{e}} \alpha \cdot \delta_{e}+C_{L}^{M a \cdot \alpha} M a \cdot \alpha+C_{L}^{M a \cdot \delta_{e}} M a \cdot \delta_{e} \\
C_{M_{y y}}=C_{M_{y y}}^{0}+C_{M_{y y}}^{\alpha} \alpha+C_{M_{y y}}^{\delta_{e}} \delta_{e}+C_{M_{y y} \alpha^{2}} \alpha^{2}+C_{M_{y y}}^{\alpha \cdot \delta_{e}} \alpha \cdot \delta_{e}+C_{M_{y y}}^{M a \cdot \alpha} M a \cdot \alpha+C_{M_{y y}}^{M a \cdot \delta_{e}} M a \cdot \delta_{e}
\end{array}\right.
$$

where $M_{a}$ is the Mach number, $\rho_{0}$ is the standard atmospheric density and $H_{0}$ is its corresponding height, and 1/ $H_{S}$ is the attenuation law of atmospheric density. $\delta_{e}$ is elevator deflection angle, and $C_{D}, C_{L}$, and $C_{M_{y y}}$ are the aerodynamic coefficients related to the angle of attack, the angle of elevator, and the Mach number, respectively.

For the separation time of hypersonic vehicle is very short, the difference between the disturbed motion parameters near the separation point and the undisturbed motion parameters is very small. At the separation time, the rolling motion and lateral motion amplitude of the aircraft are very small, and the influence on the attitude of the aircraft can be ignored. Therefore, small disturbance linearization can be used in the modeling process.

By linearizing the above equation with small disturbance, the longitudinal dynamic model of hypersonic vehicle can be obtained as follows:

$$
\begin{aligned}
& \dot{x}=A x+B u, \\
& y=C x+D u,
\end{aligned}
$$

Where $x=\left[\alpha w_{z}\right]^{T}$.

$A=\left[\begin{array}{cc}-a_{4} & 1 \\ -a_{2} & -a_{1}\end{array}\right], B=\left[\begin{array}{l}-a_{5} \\ -a_{3}\end{array}\right], C=\left[\begin{array}{ll}1 & 0 \\ 0 & 1\end{array}\right], C=\left[\begin{array}{ll}1 & 0 \\ 0 & 1\end{array}\right], D=\left[\begin{array}{l}0 \\ 0\end{array}\right]$.

Make the dynamical model as subsystem 1 when the hypersonic vehicle is in the separation interference flow field:

$$
\begin{aligned}
& \dot{x}_{1}=A_{1} x+B_{1} u, \\
& \dot{x}_{1}=A_{1} x+B_{1} u .
\end{aligned}
$$

Make the dynamical model as subsystem 2 after the hypersonic vehicle flies out of the separation interference flow field:

$$
\begin{aligned}
& \dot{x}_{2}=A_{2} x+B_{2} u, \\
& y_{2}=C_{2} x+D_{2} u,
\end{aligned}
$$




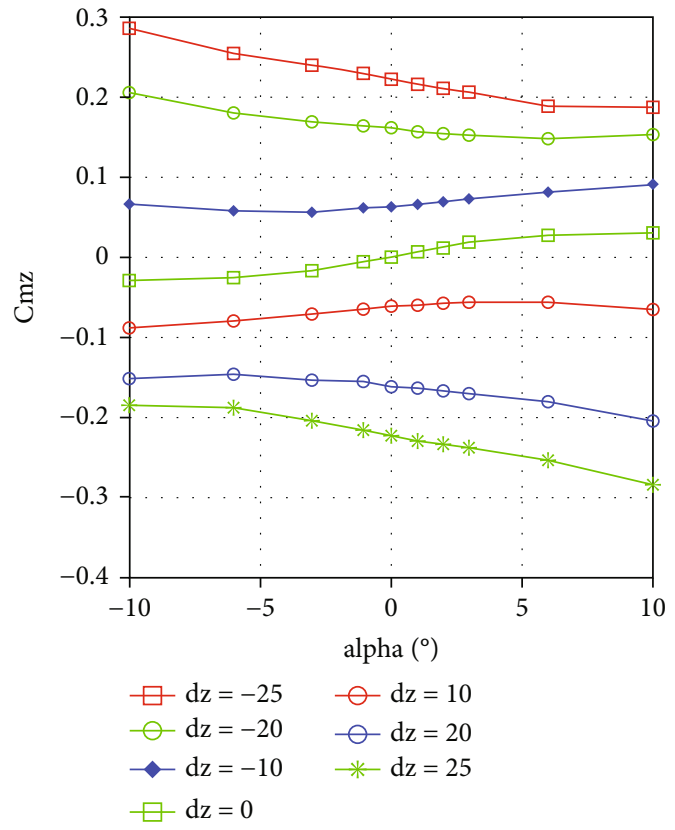

Figure 6: Variation curve of pitching moment coefficient under normal conditions.

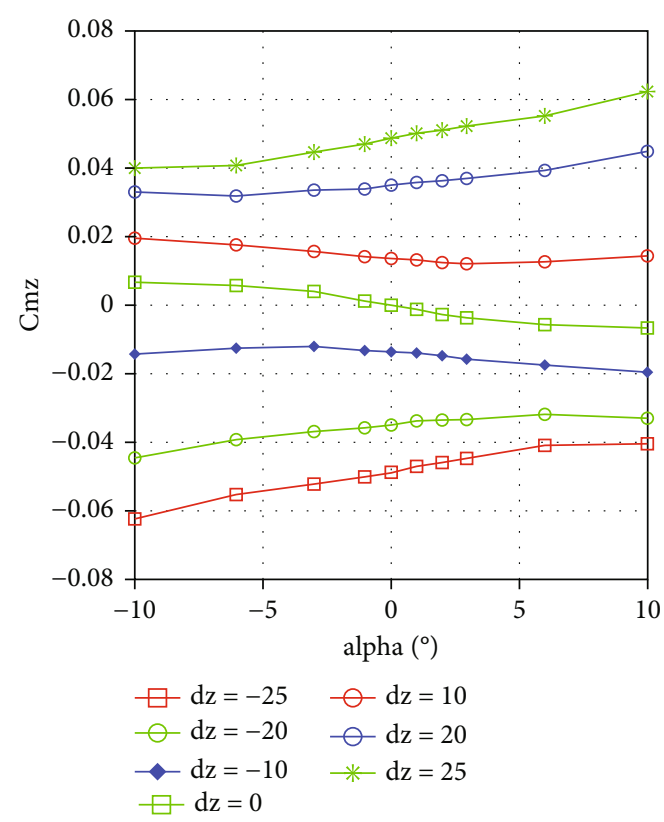

FIGURE 7: Variation curve of pitching moment coefficient under the disturbance flow field.

where $A_{1}, B_{1}$ is the coefficient matrix of the hypersonic vehicle before the separation, while $A_{2}, B_{2} A_{2}$ is the coefficient matrix of hypersonic vehicle after the separation.

2.3. Design of Sliding Mode Switching Controller for Hypersonic Stage Separation. The process of hypersonic stage separation can be regarded as switching from the subsystem in interference flow field to the subsystem out of the interference flow field. The control system is aimed at using the sliding mode switching control principle and designing a sliding mode switching controller based on a sliding switching surface, so as to realize the attitude stabilization of the hypersonic vehicle in the process of stage separation.

\subsubsection{Consider a Nonlinear System.}

$$
\begin{gathered}
\dot{x}_{(t)}=A_{\sigma_{t}} x_{(t)}+B_{\sigma_{t}} u_{\sigma_{t}}+f_{i}\left(x, \quad t, \quad \sigma_{t}\right), \\
y=C_{\sigma_{t}} x_{(t)},
\end{gathered}
$$

where $x_{(t)} \in R^{n}$ is the system's state vector, $u_{\sigma_{t}} \in R^{m}$ is the control input vector, and $y \in R^{n}$ is the output vector. $\sigma_{t}$ is the switching signal. $\sigma_{t}=I$ indicates that the $i$-th subsystem is activated.

The system can be expressed as

$$
\begin{gathered}
\dot{x}_{(t)}=A_{i} x_{(t)}+B_{i} u_{i}, \\
y=C_{i} x_{(t)} .
\end{gathered}
$$

Assumption 1. The matrix pairs $\left(A_{i,} B_{i}\right)$ and $\left(\begin{array}{ll}A_{i} & C_{i}\end{array}\right)$ are controllable and observable, respectively.

From the observability of $\left(A_{i}, C_{i}\right)$, it can be known that there is the matrix $L_{i}$ that makes $\left(A_{i}-L_{i} C_{i}\right)$ stable. Therefore, for any positive definite matrix $Q_{i}$, the following Lyapunov equation has a unique positive definite symmetric solution $P_{i}$ :

$$
\left(A_{i}-L_{i} C_{i}\right)^{T} P_{i}+P_{i}\left(A_{i}-L_{i} C_{i}\right)=-Q_{i}
$$

This paper assumes that the output matrix satisfies $C_{i}$ $=\left[\begin{array}{ll}I_{i r} & 0\end{array}\right]$, so systems (7) and (8) can be expressed as follows:

$$
\begin{gathered}
{\left[\begin{array}{c}
\dot{\bar{x}}_{1} \\
\dot{\bar{x}}_{2}
\end{array}\right]=\left[\begin{array}{cc}
A_{i 11} & A_{i 12} \\
A_{i 21} & A_{i 22}
\end{array}\right]\left[\begin{array}{l}
\bar{x}_{1} \\
\bar{x}_{2}
\end{array}\right]+\left[\begin{array}{c}
B_{i 1} \\
B_{i 2}
\end{array}\right] u_{i},} \\
y_{(t)}=\bar{x}_{1},
\end{gathered}
$$

where $\bar{x}_{1} \in R^{r}, A_{i 11} \in R^{r \times r}$, and $B_{i 1} \in R^{r \times m}$.

Assumption 2. There is a continuous function $M_{i}$ that makes $f_{i}(x, t)<B_{i} M_{i}, \quad\left\|M_{i}\right\| \leq \beta_{i} \eta_{i}, \quad$ and $\quad\left(y, \overline{x_{2}}\right)$, and $\left(y, \overline{s_{2}}(t)\right)$ satisfy $\left\|\beta_{i}-\eta_{i}\right\| \leq \mu\left\|\bar{x}_{2}(t)-\bar{s}_{2}(t)\right\|$, where $\|\cdot\|$ expresses Euclidean norm.

For systems (11) and (12), there is $K_{i}$ that makes $\left(A_{i 22}\right.$ $\left.+K_{i} A_{i 12}\right)$ stable. It can be learnt from formula (10) and $C_{i}$ $=\left[\begin{array}{ll}I_{\text {ir }} & 0\end{array}\right]$ that $K_{i}=P_{i 22}^{-1} P_{i 21}$, where

$$
\begin{gathered}
P_{i}=\left[\begin{array}{ll}
P_{i 11} & P_{i 12} \\
P_{i 21} & P_{i 22}
\end{array}\right], \\
Q_{i}=\left[\begin{array}{ll}
Q_{i 11} & Q_{i 12} \\
Q_{i 21} & Q_{i 22}
\end{array}\right] .
\end{gathered}
$$


For all $i$, the following Lyapunov equation holds:

$$
\left(A_{i 22}+P_{i 22}^{-1} P_{i 21} A_{i 12}\right)^{T} P_{i 22}+P_{i 22}\left(A_{i 22}+P_{i 22}^{-1} P_{i 21} A_{i 12}\right)=-Q_{i 22} .
$$

Construct the state observer:

$$
\begin{aligned}
\dot{\bar{z}}_{2}= & \left(A_{i 22}+P_{i 22}^{-1} P_{i 21} A_{i 12}\right) \bar{z}_{2} \\
& +\left[A_{i 21}+P_{i 22}^{-1} P_{i 21} \times A_{i 11}-\left(A_{i 22}+P_{i 22}^{-1} P_{i 21} A_{i 12}\right) P_{i 22}^{-1} P_{i 21}\right] y \\
& +\left(B_{i 2}+P_{i 22}^{-1} P_{i 21} B_{i 1}\right) u_{i},
\end{aligned}
$$

$$
\bar{s}_{2}=\bar{z}_{2}-P_{i 22}^{-1} P_{i 21} y
$$

Theorem 3. For any initial states $x(0)$ and $\bar{z}(0)$, there exists a constant $a>0$ in state observers (15) and (16) that makes $\|$ $\overline{x_{2}}(t)-\overline{s_{2}}(t) \| \leq a$, where $\overline{s_{2}}(t)$ is the 4observation state of $\overline{x_{2}}$ $(t)$.

Proof. Introduce linear transformation $z=T_{i} x$, and $T_{i}$ can be defined as

$$
T_{i}=\left[\begin{array}{cc}
I_{r} & 0 \\
P_{i 22}^{-1} P_{i 21} & I_{n-r}
\end{array}\right] .
$$

From formulas (11), (12), and (17), the following dynamic systems can be obtained:

$$
\begin{aligned}
\dot{z}_{2}= & \left(A_{i 22}+P_{i 22}^{-1} P_{i 21} A_{i 12}\right) z_{2} \\
& +\left[A_{i 21}+P_{i 22}^{-1} P_{i 21} \times A_{i 11}-\left(A_{i 22}+P_{i 22}^{-1} P_{i 21} A_{i 12}\right) P_{i 22}^{-1} P_{i 21}\right] z_{1} \\
& +\left(B_{i 2}+P_{i 22}^{-1} P_{i 21} B_{i 1}\right) u_{i},
\end{aligned}
$$

$$
y=z_{1}
$$

It can be obtained from $z=T_{i} x$ and formula (16) that $z_{2}=\overline{x_{2}}+P_{i 22}^{-1} P_{i 21} y$. According to the definition $\overline{z_{2}}=\overline{s_{2}}+P_{i 22}^{-1}$ $P_{i 21} y$, it can be obtained that $\overline{x_{2}}-\overline{s_{2}}=z_{2}-\overline{z_{2}}$. Assume the observation error $e=\overline{x_{2}}-\overline{s_{2}}$; it can be obtained from formulas (15) and (18) that

$$
\dot{e}=\left(A_{i 22}+P_{i 22}^{-1} P_{i 21} A_{i 12}\right) e .
$$

For the error system (19), consider the following Lyapunov function:

$$
V_{e}=\sum_{i=1}^{N} e^{T} P_{i 22} e .
$$

Calculate the derivative of time for $V_{e}$, we can get

$$
\begin{aligned}
V_{e} & =\sum_{i=1}^{N} e^{T}\left[\left(A_{i 22}+P_{i 22}^{-1} P_{i 21} A_{i 12}\right)^{T} P_{i 22}+P_{i 22}\left(A_{i 22}+P_{i 22}^{-1} P_{i 21} A_{i 12}\right)\right] e \\
& =-\sum_{i=1}^{N} e^{T} Q_{i 22} e<0 .
\end{aligned}
$$

According to the stability theory of Lyapunov, the error system (19) is asymptotically stable, so there is a positive constant $a$ which satisfies $\left\|x_{2}(t)-s_{2}(t)\right\| \leq a$ for the motion in any initial state.

The proof completes.

Design the sliding mode control to ensure that the state of the system reaches the sliding mode area and performs sliding mode motion. The reachable condition for the sliding state of systems (7) and (8) is

$$
V=\sum_{i=1}^{N} s_{i}^{T} W_{i} \dot{s}_{i}<0,
$$

where $W_{i}$ is symmetric positive definite matrix and $s_{i}$ is the sliding mode function of each subsystem. The definition is as follows:

$$
s_{i}=F_{i 1} y+F_{i 2} \overline{s_{2}} .
$$

In the new coordinate $(x, e)$, formula (24) can be expressed as

$$
s_{i}=F_{i 1} x-F_{i 2} e
$$

where $F_{i}=\left[\begin{array}{ll}F_{i 1} & F_{i 2}\end{array}\right]$ is the parameter to be designed and $F_{i 1} \in R^{m \times r} . F_{i}$ should satisfy that $A_{i}-B_{i}\left(F_{i} B_{i}\right)^{-1} F_{i} A_{i}$ has $n$ $-m$ eigenvalues with a negative real part; meanwhile, $F_{i} B_{i}$ is nonsingular.

The derivative of the sliding mode function obtained from formulas (20) and (25) is as follows:

$$
\dot{s}_{i}=F_{i}\left(A_{i} x+B_{i} u_{i}\right)-F_{i 2}\left(A_{i 22}+P_{i 22}^{-1} P_{i 21} A_{i 12}\right) e .
$$

When the system enters the sliding mode dynamic area, $\dot{s}_{i}=0$ can get equivalent control:

$$
u_{i e q}(t)=-\left(F_{i} B_{i}\right)^{-1}\left[F_{i} A_{i} x-F_{i 2}\left(A_{i 22}+P_{i 22}^{-1} P_{i 21} A_{i 12}\right) e\right] .
$$

Express the formula $\dot{V}$ as follows:

$$
\dot{V}=\sum_{i=1}^{N}\left(W_{i} s_{i}\right)^{T} \overline{u_{i}} .
$$


Make $\overline{u_{i}}=-m_{i} s_{i}$, and $m_{i}>0$. The definition is:

$$
\overline{u_{i}}=\left\{\begin{array}{l}
-m_{i} \frac{W_{i} s_{i}}{\left\|W_{i} s_{i}\right\|}, \\
0, \\
s_{i} \neq 0 .
\end{array}\right.
$$

The equivalent control $u_{\text {ieq }}$ satisfies that $s_{i}=0$; therefore,

$$
F_{i}\left(A_{i} x+B_{i} u_{i e q}\right)-F_{i 2}\left(A_{i 22}+P_{i 22}^{-1} P_{i 21} A_{i 12}\right) e=0 .
$$

Subtracting formula (30) from formula (26), it can obtain

$$
\dot{s}_{i}=F_{i} B_{i}\left(u_{i}-u_{i e q}\right)=0 .
$$

Putting formula (27) into formula (31), we can get

$$
u_{i}=-\left(F_{i} B_{i}\right)^{-1}\left[F_{i} A_{i} x-\left(A_{i 22}+P_{i 22}^{-1} P_{i 21} A_{i 12}\right) e-\overline{u_{i}}\right]
$$

Formula (32) needs to change its form as it contains the nonlateral state vector $\overline{x_{2}}$.

Theorem 4. Consider the definition controller of switching systems (7) and (8):

$$
\begin{aligned}
u_{i}= & -\left(F_{i} B_{i}\right)^{-1}\left[\left(F_{i 1} A_{i 12}+F_{i 2} A_{i 22}\right) y\right. \\
& \left.+\left(F_{i 1} A_{i 12}+F_{i 2} A_{i 22}\right) \overline{s_{2}}+K_{i}(y, t)+\left\|F_{i} B_{i}\right\| \beta_{i} \eta_{i}-\overline{u_{i}}\right],
\end{aligned}
$$

where $\overline{u_{i}}$ can be defined as formula (29).

$$
K_{i}>a\left(\left\|F_{i 1} A_{i 12}-F_{i 2} P_{i 22}^{-1} P_{i 21} A_{i 12}\right\|+\beta_{i} \eta_{i}\left\|F_{i} B_{i}\right\|\right) .
$$

Under the effect of the control (33), systems (7) and (8) enter the sliding mode dynamic zone and perform sliding mode motion.

Proof. When $s_{i}=0$, the system has entered the sliding mode area for sliding mode motion. In the case of $s_{i} \neq 0$, substitute formula (33) into formula (26) and formula (23) gives

$$
\begin{aligned}
V= & \sum_{i=1}^{N} s_{i}^{T} W_{i}\left[F_{i} A_{i} x-\left(F_{i 1} A_{i 11}+F_{i 2} A_{i 21}\right) y\right. \\
& -\left(F_{i 1} A_{i 12}+F_{i 2} A_{i 22}\right) \overline{s_{2}}+\overline{u_{i}}-\left\|F_{i} B_{i}\right\| \beta_{i} \eta_{i}-K_{i}(t) \\
& \left.-F_{i 2}\left(A_{i 22}+P_{i 22}^{-1} P_{i 21} A_{i 12}\right) e\right] .
\end{aligned}
$$

After simplification, we can know that

$$
\begin{aligned}
F_{i} A_{i} x- & \left(F_{i 1} A_{i 11}+F_{i 2} A_{i 21}\right) y-\left(F_{i 1} A_{i 12}+F_{i 2} A_{i 22}\right) \overline{s_{2}} \\
& =\left(F_{i 1} A_{i 12}+F_{i 2} A_{i 22}\right) e .
\end{aligned}
$$

It can be known from Assumption 2 that

$$
\begin{gathered}
F_{i} f_{i}-\left\|F_{i} B_{i}\right\| \beta_{i} \eta_{i}=F_{i} B_{i} M_{i}(t)-\left\|F_{i} B_{i}\right\| \leq\left\|F_{i} B_{i}\right\|\left\|M_{i}\right\| \beta_{i} \eta_{i} \\
\leq \beta_{i} \eta_{i}\left\|F_{i} B_{i}\right\|\|e\| .
\end{gathered}
$$

Substituting formulas (36) and (37) into formula (35), we can get

$$
\begin{aligned}
V= & \sum_{i=1}^{N} s_{i}^{T} W_{i}\left[\overline{u_{i}}+\left(F_{i 1} A_{i 12}+F_{i 2} P_{i 22}^{-1} P_{i 21} A_{i 12}\right) e\right. \\
& \left.+\beta_{i} \eta_{i}\left\|F_{i} B_{i}\right\|\|e\|-K_{i}(t)\right] \\
\leq & \sum_{i=1}^{N} s_{i}^{T} W_{i}\left[\overline{u_{i}}+a\left(\left\|F_{i 1} A_{i 12}+F_{i 2} P_{i 22}^{-1} P_{i 21} A_{i 12}\right\|\|e\|+\beta_{i} \eta_{i}\left\|F_{i} B_{i}\right\|\|e\|\right)\right. \\
& \left.-K_{i}(t)\right]<\sum_{i=1}^{N} s_{i}^{T} W_{i} \overline{u_{i}}<-\sum_{i=1}^{N} m_{i} s_{i}^{T} W_{i} \frac{W_{i} s_{i}}{\left\|W_{i} s_{i}\right\|}<0 .
\end{aligned}
$$

Therefore, the sliding mode arrival conditions are satisfied, and under the action of control (33) and a given switching rate, systems (7) and (8) can enter the sliding mode area and perform sliding mode motion.

The proof completes

\section{Results and Discussion}

3.1. Simulation Verification. Assume that the hypersonic vehicle flies at an altitude of $19 \mathrm{~km}$ with the flying speed of 6.0 Ma. When stage separating, the initial attack angle $\alpha=$ $0^{\circ}$, and the initial angular velocity $w_{z}=1^{\circ} / \mathrm{s}$. During the stage separation, systems of the hypersonic vehicle in and out of the interference flow field are subsystem 1 and subsystem 2 , respectively, switching from subsystem 1 to subsystem 2 at $200 \mathrm{~mm}$ seconds. Only considering the antieffect of the surface control without regard to the interference in the separation process, the dynamic system matrix in the process of stage separation can be calculated as follows:

$$
\begin{gathered}
A_{1}=\left[\begin{array}{cc}
-0.3563 & 23.7567 \\
1 & -0.0955
\end{array}\right], A_{2}=\left[\begin{array}{cc}
-0.3632 & 9.2644 \\
1 & -0.2039
\end{array}\right], \\
B_{1}=\left[\begin{array}{c}
-25.4341 \\
0
\end{array}\right], B_{2}=\left[\begin{array}{c}
-74.0901 \\
-0.0355
\end{array}\right], \\
Q_{1}=Q_{2}=6 I_{2} .
\end{gathered}
$$

Let $L_{1}=\left[\begin{array}{ll}12 & 4\end{array}\right], L_{2}=\left[\begin{array}{ll}14 & 2\end{array}\right]$; solving formula (10) gives a positive definite symmetric matrix:

$$
P_{1}=\left[\begin{array}{cc}
0.2709 & -0.1157 \\
-0.1157 & 2.6255
\end{array}\right], P_{2}=\left[\begin{array}{cc}
0.2235 & -0.2107 \\
-0.2107 & 5.1401
\end{array}\right] \text {. }
$$

The coefficient matrix $F_{1}=\left[\begin{array}{ll}5 & 3\end{array}\right], F_{2}=\left[\begin{array}{ll}6 & 5\end{array}\right]$ can be 


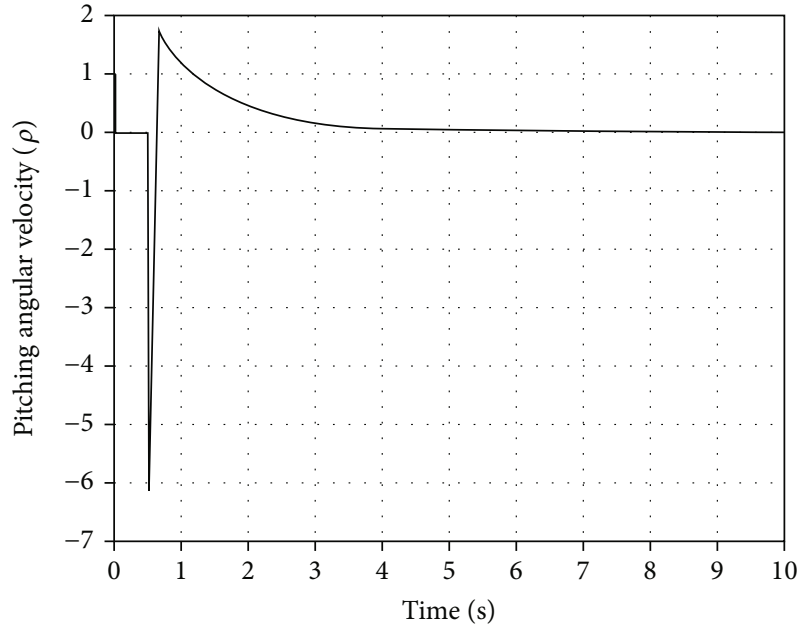

FIGURE 8: State response graph of pitching angular velocity.

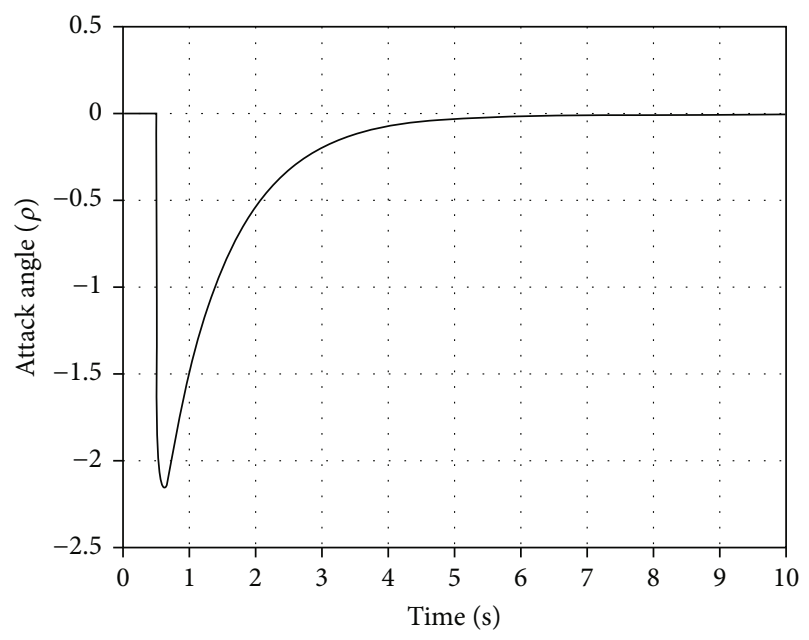

Figure 9: State response graph of attack angle.

obtained according to the requirements of $F_{i}$, and response diagrams of pitch angle and attack angle are shown as Figures 8, 9.

Assume that the hypersonic vehicle is also affected by the interference torque during the separation process, whose form of interference is as follows:

$$
f_{1}=B_{1} M_{1}(y)=B_{1} \sin x_{1}, f_{2}=B_{2} M_{2}(y)=B_{2} \sin x_{2} .
$$

The interference satisfies

$$
\begin{gathered}
\left\|M_{1}\right\| \leq\left|x_{1}\right|,|| M_{2} \| \leq\left|x_{2}\right|, \\
\beta_{1}=\beta_{2}=1, \eta_{1}=\left|x_{1}\right|, \eta_{2}=\left|x_{2}\right| .
\end{gathered}
$$

Let $K_{1}=K_{2}=1$, and the state response diagrams are shown in Figures 10, 11.

3.2. Discussion. Figure 12 shows the response curve of the conventional controller when there is stage separation interference. At the beginning of separation, the aircraft precursor is in the interference flow field; the control surface

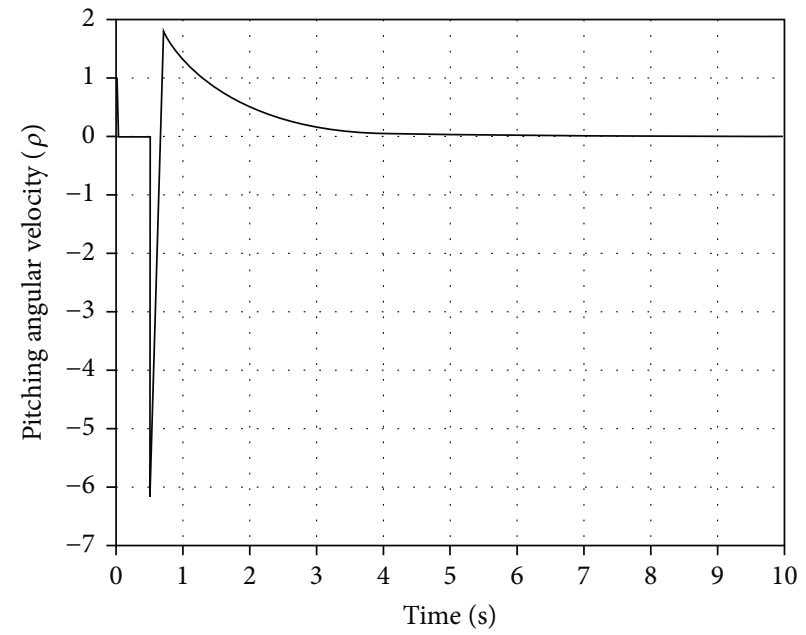

FIGURE 10: State response graph of pitching angular velocity.

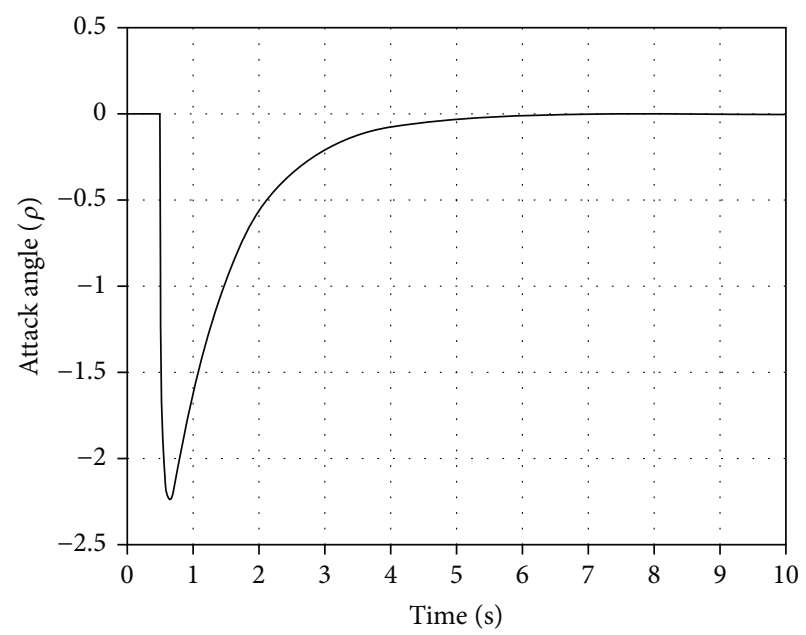

FIGURE 11: State response graph of attack angle.

shows reversal effect. The angular velocity increases with the increase of rudder deflection angle until the rudder surface is saturated. At this time, the angular rate continues to increase under the influence of interference; after the front body of flight is out of the interference area, the rudder surface is not saturated, producing the suppression effect on interference. Therefore, the control system is at risk of losing control when using a single-mode control method. While adopting the switching control based on sliding mode, the control system switches the control mode according to the state during separation, which can effectively suppress the separation interference and prevent the antimanipulation of the rudder surface.

It can be seen from the simulation results shown in Figures 8-11 that when the flow field interference during stage separation makes the rudder surface ineffective, sliding mode switching control can not only realize reliable separation of the hypersonic aircraft from the booster under a high dynamic pressure but also quickly suppress the interference of the separation process on the vehicle attitude. Under the 


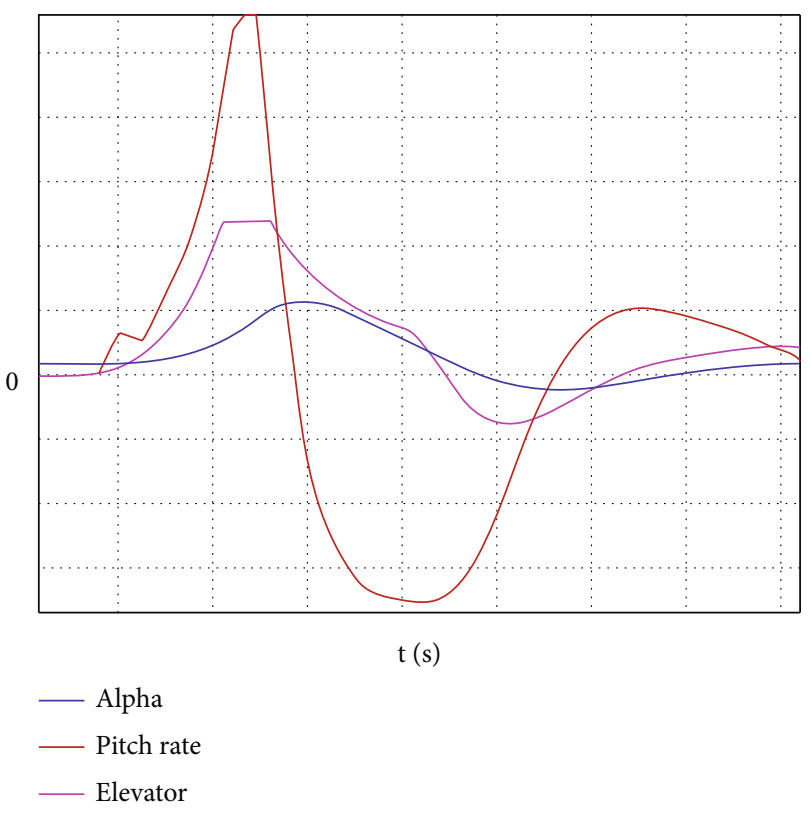

FIGURE 12: Response of conventional controller under interference.

action of interference, the designed controller can converge the angle of attack response of the aircraft to the equilibrium angle of attack within 5 seconds, and the amplitude is no more than $5^{\circ}$. The pitch angular velocity converges within 3 seconds, and the amplitude does not exceed $6 \%$ s. The dynamic characteristics of the system meet the design requirements.

\section{Conclusion}

Aiming at the interference suppression of hypersonic stage separation under a high dynamic pressure, this paper proposes a method of hypersonic stage separation control based on sliding mode switching control. The flight state of the hypersonic separation process in and out of the interference flow field region is regarded as a subsystem with switching characteristics. A sliding mode switching control system is designed to realize the attitude stabilization in different states during the stage separation process. Simulation results show that the designed control system can realize the reliable stage separation under a high dynamic pressure and make the effective suppression on the interference in the separation process, with the advantages of stability and rapidity.

\section{Data Availability}

Data sharing not applicable to this article as no datasets were generated or analyzed during the current study.

\section{Conflicts of Interest}

The authors declared no potential conflicts of interest with respect to the research, authorship, and/or publication of this article.

\section{References}

[1] H. An, Q. Wu, H. Xia, and C. Wang, "Multiple Lyapunov function-based longitudinal maneuver control of airbreathing hypersonic vehicles," International Journal of Control, vol. 94, no. 2, pp. 286-299, 2021.

[2] H. An, Q. Wu, H. Xia, C. Wang, and X. Cao, "Adaptive controller design for a switched model of air-breathing hypersonic vehicles," Nonlinear Dynamics, vol. 94, no. 3, pp. 1851-1866, 2018.

[3] P. G. Buning, T. C. Wong, A. D. Dilley, and J. L. Pao, "Computational fluid dynamics prediction of hyper-X stage separation aerodynamics," Journal of Spacecraft and Rockets, vol. 38, no. 6, pp. 820-827, 2001.

[4] W. C. Woods, S. D. Holland, and M. DiFulvio, "Hyper-X Stage separation wind-tunnel test program," Journal of Spacecraft and Rochets, vol. 38, no. 6, pp. 811-819, 2001.

[5] P. R. Sudalagunta, C. Sultan, R. K. Kapania, L. T. Watson, and P. Raj, "Aeroelastic control-oriented modeling of an airbreathing hypersonic vehicle," Journal of Guidance, Control, and Dynamics, vol. 41, no. 5, pp. 1136-1149, 2018.

[6] Y. Shengjiang and L. Chaoyi, "Research on stage separation control technology of hypersonic aircraft in the United States," Flight Missile, vol. 11, pp. 34-42, 2014.

[7] Q. Li Yongtao and T. W. Xiangju, "Dynamics modeling and simulation of air-breathing hypersonic flight aircraft," Flight Mechanics, vol. 32, no. 5, pp. 403-407, 2014.

[8] W. Xun, L. Jun, and X. Zhixun, "Numerical simulation of booster separation for an air-breathing hypersonic vehicle," Journal of National University of Defense Technology, vol. 41, no. 1, pp. 34-40, 2019.

[9] Y. Li, B. Reimann, and T. Eggers, "Coupled simulation of CFD-flight-mechanics with a two-species-gas-model for the hot rocket staging," Acta Astronautica, vol. 128, pp. 44-61, 2016.

[10] Y. Binbin, M. Zhongjie, W. Xin, and Y. Jie, "An effective rudder deflection presetting design method for suppressing aerodynamic interference of hypersonic stage separation," Journal of Northwestern Polytechnical University, vol. 29, no. 5, pp. 757-760, 2011.

[11] L. Wu and J. Lam, "Sliding mode control of switched hybrid systems with time-varying delay," International Journal of Adaptive Control and Signal Processing, vol. 22, no. 10, pp. 909-931, 2008.

[12] L. Wu, D. W. C. Ho, and C. W. Li, "Sliding mode control of switched hybrid systems with stochastic perturbation," Systems Control Letters, vol. 60, no. 8, pp. 531-539, 2011.

[13] X. Yu, C. Wu, F. Liu, and L. Wu, "Sliding mode control of discrete-time switched systems with time-delay," Journal of the Franklin Institute, vol. 350, no. 1, pp. 19-33, 2013. 